\title{
Comportamiento, para el terremoto de Lorca de 11-05-2011, de edificios de vigas planas proyectados sin tener en cuenta la acción sísmica
}

\author{
Behavior, for the Lorca earthquake on 11-05-2011, of wide beam building \\ designed without seismic considerations
}

D. Domínguez ${ }^{(*)}, \underline{\text { F. López-Almansa }}^{(* *)}$, A. Benavent-Climent ${ }^{(* *)}$

\begin{abstract}
RESUMEN
El terremoto de Lorca (11-05-2011) fue el movimiento sísmico más destructivo registrado en España, a pesar de su moderada magnitud. Este artículo describe la simulación numérica de la respuesta dinámica, al registro principal del terremoto de Lorca, de seis edificios de tres y seis plantas con forjados unidireccionales de hormigón con vigas planas; estos edificios fueron proyectados sin tener en cuenta la acción sísmica. Se ha elegido esta tipología constructiva por presentar, potencialmente, una elevada vulnerabilidad sísmica. Los seis edificios han sido seleccionados para representar un número importante de edificios de este tipo existentes en zonas de sismicidad baja o media (como Lorca) de España y correspondientes a los años 1974-1994, posteriores a la PDS-1 1974 y previos a la NCSE-94. Los resultados obtenidos muestran que estos edificios, aun contando con la cooperación de los muros, no poseen capacidad para resistir la componente más intensa del registro de Lorca.
\end{abstract}

Palabras clave: Vigas planas; terremoto de Lorca; vulnerabilidad sísmica; respuesta dinámica; colapso.

\section{SUMMARY}

The recent earthquake in Lorca (11-05-2011) was the most destructive recorded event in Spain, despite its moderate magnitude. This paper describes the numerical simulation of the dynamic response to the main record of the Lorca earthquake of six 3 and 6-story buildings with one-way concrete slabs with wide beams; these buildings were designed without any seismic consideration. We have chosen this type of construction because it is potentially highly vulnerable. The six considered buildings were selected to represent a large number of buildings of this type in areas of low-to-medium seismicity (as Lorca) of Spain along the period 1974-1994. The results show that these buildings, even with the cooperation of the walls, do not have sufficient capacity to withstand the most severe component of the Lorca record.

Keywords: Wide beams; Lorca earthquake; seismic vulnerability; time history; collapse.

(*) Universidad Internacional de Cataluña, Barcelona (España).

(**) Universidad Politécnica de Cataluña, Barcelona (España).

(***) Universidad de Granada, (España).

Persona de contacto/Corresponding author: francesc.lopez-almansa@upc.edu (F. López-Almansa)

Cómo citar este artículo/Citation: Domínguez, D., López-Almansa, F., Benavent, A. (2014). Comportamiento, para el terremoto de Lorca de 11-05-2011, de edificios de vigas planas proyectados sin tener en cuenta la acción sísmica. Informes de la Construcción, 66(533): eoo8, doi: http://dx.doi.org/10.3989/ic.12.092.

Licencia / License: Salvo indicación contraria, todos los contenidos de la edición electrónica de Informes de la Construcción se distribuyen bajo una licencia de uso y distribución Creative Commons Reconocimiento no Comercial 3.o. España (cc-by-nc). 


\section{INTRODUCCIÓN}

El terremoto de Lorca representó probablemente uno de los mayores hitos en la historia de la ingeniería sísmica en España ya que, desgraciadamente, puso de manifiesto tanto la vulnerabilidad de muchas construcciones como la relativamente inesperada virulencia de la acción sísmica. Este trabajo pretende contribuir a establecer el nivel de inseguridad, frente a movimientos sísmicos de severidad comparable a la del terremoto de Lorca, de edificios con forjados unidireccionales de hormigón con vigas planas y correspondientes al período de vigencia de la antigua normativa sismorresistente (1) (1974-1994). Se ha elegido a este tipo de edificaciones por su abundancia en España y porque, potencialmente, pueden presentar una elevada vulnerabilidad sísmica (2); la época considerada representa un período en que la normativa vigente planteaba unas exigencias excesivamente laxas. A partir de los resultados de este trabajo y de otros estudios, la investigación de los autores se centra actualmente en proponer estrategias de rehabilitación sísmica para edificios de vigas planas.

Los edificios de vigas planas se caracterizan por poseer estructura de hormigón armado con forjados unidireccionales de canto constante; en consecuencia, los encofrados son lisos y la superficie inferior de los forjados es plana, permitiendo pasar libremente las instalaciones. A causa de estas ventajas, estas soluciones estructurales han sido y son bastante utilizadas en España (y en otros países de su entorno), aun en las zonas de mayor sismicidad. En la dirección de los pórticos (es decir, de las vigas planas) el comportamiento sismorresistente de este tipo de edificios ofrece pocas garantías ya que (i) la resistencia y la rigidez lateral de los edificios es reducida, (ii) la ductilidad de las vigas es baja por la elevada cuantía de las armaduras, (iii) las compresiones en las bielas que se forman en el interior de los nudos pueden ser excesivas debido a su elevada inclinación con respecto a la vertical y (iv) la transmisión de momentos flectores entre las vigas planas y los pilares es problemática porque parte de las armaduras longitudinales de aquéllas discurren fuera del ancho de los pilares, generándose mecanismos frágiles de torsión para los que no existe suficiente armadura. En la dirección transversal la situación es aun más crítica ya que (i) los pórticos son menos resistentes y (ii) el comportamiento de los nudos entre las viguetas y las vigas es cuestionable, presentándose problemas de anclaje de las armaduras inferiores si se produce inversión de momentos. Estas carencias hacen que los análisis de resistencia sísmica sean efectuados contando con la cooperación de los muros de relleno; al menos de aquéllos construidos con ladrillos perforados (3). Dada la importante variedad de edificios de vigas planas existentes, este estudio se centra en seis edificios tipo seleccionados para representar la mayor parte de los existentes. Respecto de los muros de relleno, la variedad es aun mayor, tanto en los tipos

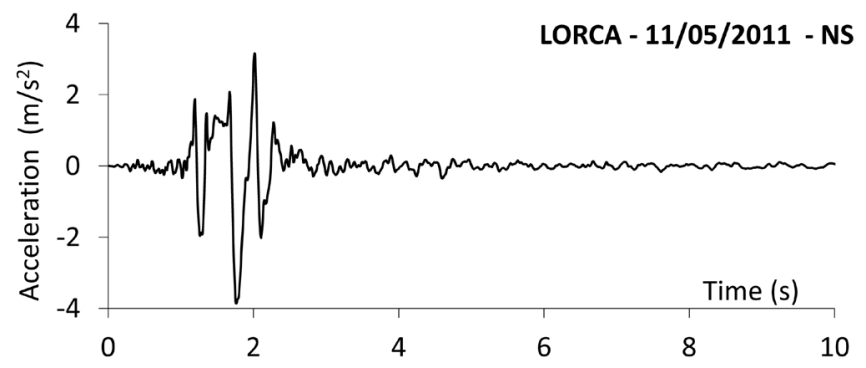

(a) Componente Norte-Sur (NS) de ladrillo como en la densidad y ubicación; por dicha razón, se consideran tres situaciones, las cuales se denotan en este artículo como "sin muros", "muros mín.” y "muros máx.”.

El comportamiento sismorresistente de los edificios tipo para el terremoto de Lorca se analiza determinando su respuesta dinámica no lineal a los acelerogramas más intensos registrados para dicho terremoto. Los desplazamientos máximos obtenidos se comparan con los desplazamientos admisibles de los edificios, determinados éstos a partir de sus curvas de capacidad, las cuales fueron determinadas en otro trabajo previo (2) mediante análisis push-over. El comportamiento dinámico de los edificios se determina mediante simulación numérica a partir de modelos cuyos parámetros se eligen según resultados experimentales obtenidos, entre otros, por algunos de los autores (4). La observación de los resultados obtenidos permite concluir que estos edificios, aun contando con la cooperación de los muros, no poseen capacidad suficiente para resistir la componente más intensa del registro de Lorca y presentan una clara situación de colapso en los instantes de mayor aceleración.

\section{TERREMOTO DE LORCA}

El terremoto de Lorca constituye uno de los movimientos sísmicos con mayor poder destructivo registrados en España (5), a pesar de su moderada magnitud $\left(M_{\mathrm{w}}=5,1\right.$; (6)); esta severidad parece deberse a la escasa profundidad hipocentral (se estima en unos $2 \mathrm{~km}$ ) y a la casi nula distancia entre el epicentro y el centro de la ciudad de Lorca (2,9 km hasta la estación sismológica). El terremoto dejó 9 víctimas mortales, 167 heridos (3 graves), 40000 personas sin hogar y causó importantes daños materiales, aún por cuantificar. La Figura 1 representa los acelerogramas de las componentes horizontales del registro más severo del acelerograma de Lorca (7).

La Figura 1 muestra que los registros del acelerograma de Lorca poseen un carácter marcadamente impulsivo, particularmente la componente norte-sur (2), del cual emana buena parte de su peligrosidad. Para la componente norte-sur el índice adimensional de Manfredi toma el valor $I_{\mathrm{D}}=2,57$ (2); el índice $I_{\mathrm{D}}$ se define como el cociente entre la integral del cuadrado de la aceleración a lo largo de la duración total y el producto de los valores máximos de la aceleración y de la velocidad. Este índice cuantifica la presencia de pulsos de velocidad, es decir, de registros impulsivos con efectos de directividad generados por la proximidad a la falla. Habitualmente se considera que valores de $I_{\mathrm{D}}$ inferiores a 10 corresponden a registros impulsivos, es decir con efectos de proximidad de falla.

La Figura 2 representa los espectros de respuesta (de aceleración absoluta $S_{\mathrm{a}}$ ) de los acelerogramas de la Figura 1 (2).

Figura 1. Acelerogramas del terremoto de Lorca (11 de mayo de 2011) (2). 
A efectos de comparación, se muestra también el espectro de respuesta de la norma española (8) para la aceleración sísmica de Lorca $\left(a_{\mathrm{b}}=0,12 \mathrm{~g}\right)$ y para suelo duro (tipo I según la NCSE-02); se aprecia inmediatamente que ambas componentes (especialmente la norte-sur) exceden claramente las prescripciones de la NCSE-02 para casi todos los períodos de interés.

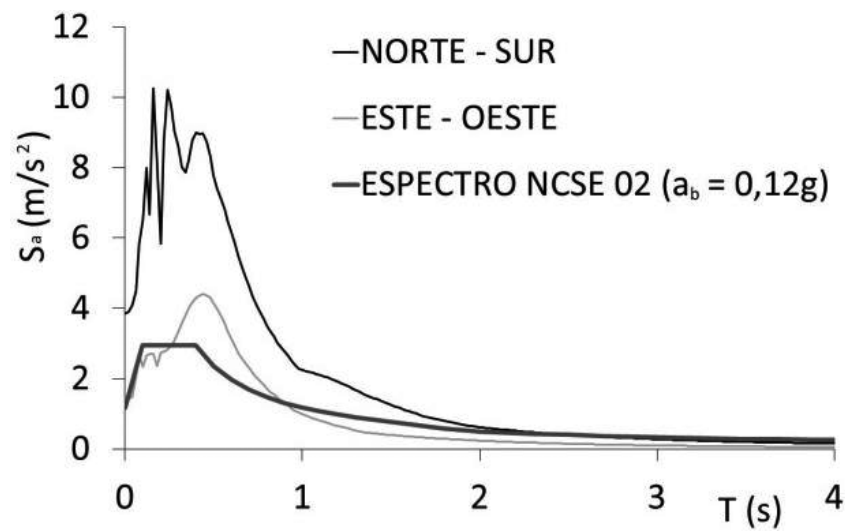

Figura 2. Espectros de respuesta del acelerograma del terremoto de Lorca. (Domínguez 2012).

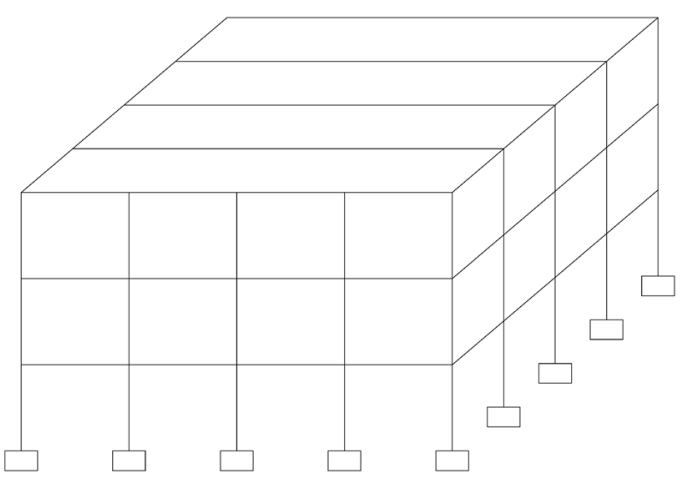

(a) Edificios de tres plantas

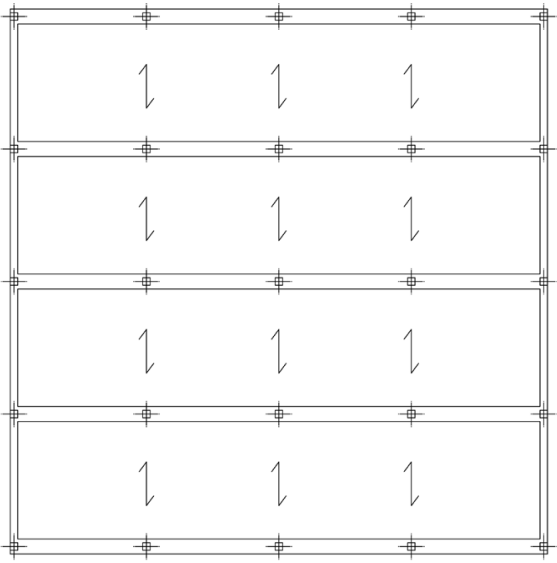

(c) Planta de un edificio tipo
La importante severidad del terremoto de Lorca hace que la verificación de la resistencia de unos edificios que son bastante abundantes en nuestro país a dicho movimiento sísmico sea un tema del mayor interés.

\section{EDIFICIOS DE VIGAS PLANAS EN ESPAÑA}

Tal como se describe en la Introducción, los edificios de vigas planas son bastante comunes en España, incluso en las zonas de mayor sismicidad. Han sido utilizados, por facilidad de construcción y de disposición de instalaciones, especialmente para uso residencial, administrativo y de pequeño comercio. Aparte de las limitaciones de la normativa sismorresistente (1) vigente en la época abarcada (1974-1994), con excesiva frecuencia ésta no era suficientemente tomada en consideración o simplemente era completamente ignorada. Esta circunstancia, unida a la alta vulnerabilidad sísmica inherente a estas construcciones (discutida previamente en la Introducción), hace que sea necesario valorar el nivel de riesgo sísmico acumulado; éste corresponde a la conjunción de la amenaza y de la vulnerabilidad, este trabajo se centra en ésta última.

Tal como se describe en la Introducción, las edificaciones de vigas planas existentes en España se representan me-

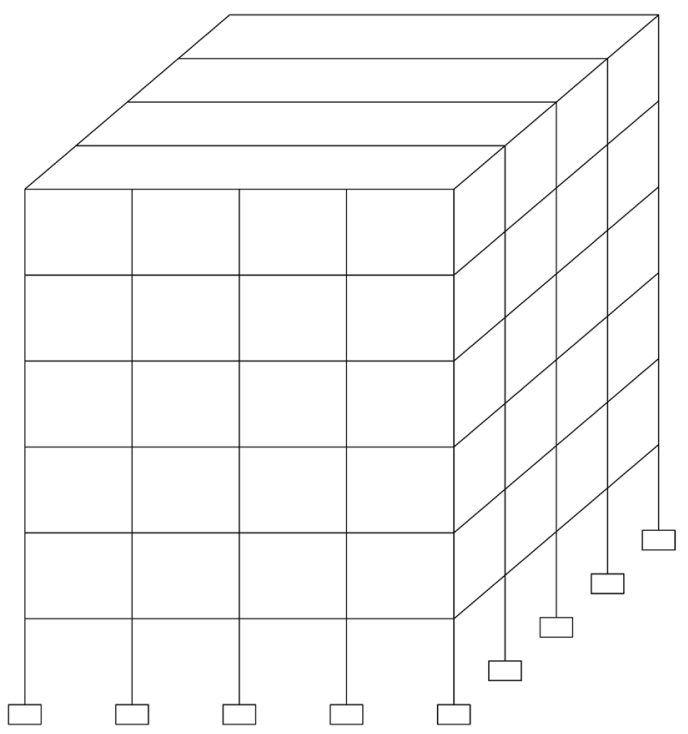

(b) Edificios de seis plantas

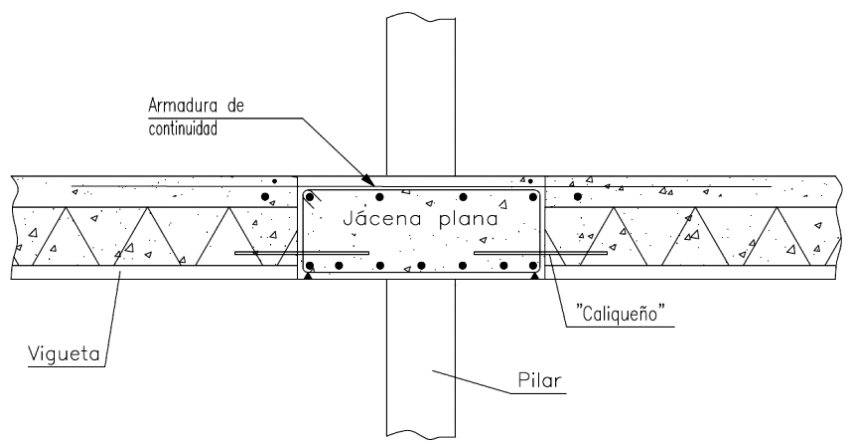

(d) Nudo pilar-viga plana

Figura 3. Espectros tipo de tres y de seis plantas. 
diante seis edificios tipo de tres y de seis plantas. Sus características generales se describen en la Figura 3; la altura de las plantas es $4 \mathrm{~m}$ en la planta baja y $3 \mathrm{~m}$ en las plantas superiores. La Figura 3a, la Figura 3b y la Figura 3c muestran que los edificios tienen planta rectangular (cuadrada, de hecho) y que su configuración es regular y simétrica; en particular, en la Figura $3 \mathrm{c}$ se aprecia que en dirección transversal (perpendicular a los pórticos) existen vigas en las fachadas, su ancho es igual al de los pilares. La Figura 3 d representa un detalle de un nudo entre un pilar y una viga plana; se muestra que las "barras de solape" garantizan la continuidad entre las viguetas para momentos negativos. La Figura 3d también señala que, de acuerdo con una práctica constructiva relativamente habitual en la época abarcada por este estudio (1974-1994), no se dispusieron armaduras en la capa superior de hormigón (de $4 \mathrm{~cm}$ de espesor), a pesar de ser éstas obligatorias. En la Figura 3d se dibujan "viguetas armadas de zapatilla", también se consideraron otras alternativas como viguetas armadas y pretensadas; en todos los casos la separación entre ejes de viguetas es de 60 $\mathrm{cm}$. A pesar de existir edificios de vigas planas con configuraciones marcadamente asimétricas, su relativa escasez y la falta de patrones comunes claros que permitan seleccionar fácilmente edificios tipo representativos, hace que éstos no sean considerados en este estudio. Por otra parte, buena parte de los edificios asimétricos corresponden a construcciones entre medianeras y la aplicabilidad de este estudio a edificios "completamente encajados entre medianeras" es cuestionable dado que, en dicho caso, el modelo a analizar debe involucrar preferentemente, a la fachada completa ya que el comportamiento sísmico de ésta es básicamente solidario.

Las características más relevantes de los edificios tipo a efectos de su comportamiento sismorresistente se describen en la Tabla 1 adjunta. Cada edificio se denota (primera columna de la Tabla 1) por dos números y un símbolo; el primer número indica el número de plantas (segunda columna de la Tabla 1), el segundo número representa la luz en $m$ (igual en ambas direcciones, tercera columna de la Tabla 1) y el símbolo especifica si las secciones de los pilares son cuadradas (indicado mediante $\square$ ) o rectangulares (indicado mediante , en este caso la dimensión larga de los pilares se dispone en el plano de los pórticos). En los edificios de $5 \mathrm{~m}$ de luz la dimensión en planta es $20 \times 20 \mathrm{~m}^{2}$ y en los edificios de 5,5 $\mathrm{m}$ de luz la dimensión en planta es $22 \times 22 \mathrm{~m}^{2}$. En los edificios de seis plantas los pilares cuadrados tienen $30 \mathrm{~cm}$ de lado en las dos plantas superiores y $10 \mathrm{~cm}$ más cada dos plantas, es decir $40 \mathrm{~cm}$ en las plantas tercera y cuarta y $50 \mathrm{~cm}$ en las plantas primera y segunda. En los edificios de seis plantas los pilares rectangulares tienen $30 \times 40 \mathrm{~cm}$ en las dos plantas superiores, $40 \times 50 \mathrm{~cm}$ en las plantas tercera y cuarta y $50 \times 60 \mathrm{~cm}$ en las plantas primera y segunda; los lados de mayor longitud pertenecen a los planos de los pórticos. La resistencia característica del hormigón es $f_{\mathrm{ck}}=175 \mathrm{~kg} / \mathrm{cm}^{2}$ y el límite elástico del acero es $f_{\mathrm{yk}}=4100 \mathrm{~kg} / \mathrm{cm}^{2}$ (AEH 400$)$.

En este tipo de construcciones la cooperación estructural de los muros debe ser considerada (9). Tal como se discute en la Introducción, la disposición de muros de relleno (tabiques, particiones, cerramientos y muros de cajas de escaleras y de ascensores) es bastante dispersa tanto en su distribución en planta como en su vinculación a la estructura principal y en los materiales utilizados (básicamente, mampostería sin armar de ladrillos perforados y huecos (3). Respecto a los ladrillos empleados, se considera que la colaboración de los ladrillos huecos debe ser despreciada (10); se cuenta sólo con la cooperación de los muros con ladrillos perforados ya que la resistencia de los ladrillos huecos no es suficientemente fiable porque esta obra de fábrica es demasiado frágil para absorber sin daño las deformaciones necesarias para movilizar la resistencia plástica imprescindible para cooperar con la estructura principal. Dada esta importante heterogeneidad, en este trabajo se han considerado tres situaciones tipo: ausencia de muros ("sin muros"), baja densidad de muros ("muros mín.") y alta densidad de muros ("muros máx."); la Figura 4 describe la ubicación de los muros en estas dos últimas situaciones. El caso "sin muros" puede corresponder a la no existencia de muros, a su extremadamente baja densidad, a muros con ladrillos huecos o a muros separados de la estructura principal. Los muros considerados tienen $15 \mathrm{~cm}$ de espesor, de los que $12 \mathrm{~cm}$ corresponden a los ladrillos y $3 \mathrm{~cm}$ al revestimiento de yeso. Se considera que existen los mismos muros en todas las plantas; no obstante, debe indicarse que en la mayoría de los edificios de viviendas, la compartimentación de la planta baja con uso comercial es mucho menor que la de las plantas superiores destinadas a las viviendas. En cada planta, se supone que los muros ocupan toda la altura libre de ésta, dado que no se tiene en cuenta el posible efecto de columna corta que se podría generar en los pilares, por el efecto de los tabiques que no llegan a la planta superior (para permitir el paso de las instalaciones).

Las armaduras se diseñan siguiendo las prescripciones de la normativa vigente en el período abarcado en este estudio; ya que éste transcurre desde 1974 a 1994, se consideran las normas EH-73, EH-8o y EH-91. La sobrecarga de uso es $2 \mathrm{kN} /$ $\mathrm{m}^{2}$ en las plantas y $1 \mathrm{kN} / \mathrm{m}^{2}$ en la cubierta. De acuerdo con la discusión previa acerca del grado de cumplimiento de la normativa sismorresistente, ésta no se toma en consideración. Obviamente, en el dimensionamiento de las armaduras no se toma en consideración la existencia de muros de relleno.

Tabla 1.

Edificios tipo

\begin{tabular}{|c|c|c|c|c|c|c|c|}
\hline Edificio & $\begin{array}{c}\text { Plantas / } \\
\text { altura (m) }\end{array}$ & Luces (m) & $\begin{array}{c}\text { Pilares planta } \\
\text { baja (cm) }\end{array}$ & $\begin{array}{c}\text { Pilares planta } \\
\text { superior (cm) }\end{array}$ & $\begin{array}{c}\text { Vigas planas } \\
(\mathbf{c m})\end{array}$ & $\begin{array}{c}\text { Período } \\
\text { fundamental (s) }\end{array}$ & $\begin{array}{c}\text { Peso } \\
(\boldsymbol{G}+\mathbf{o , 3} \mathbf{Q})(\mathbf{k N})\end{array}$ \\
\hline $3-5-\mathbf{\square}$ & $3 / 10$ & $5 \times 5$ & $40 \times 40$ & $30 \times 30$ & $25 \times 60$ & $0,585 / 1,037$ & 9770 \\
\hline $3-5,5-\boldsymbol{\square}$ & $3 / 10$ & $5,5 \times 5,5$ & $40 \times 40$ & $30 \times 30$ & $29 \times 75$ & $0,783 / 1,309$ & 10825 \\
\hline $6-5-\mathbf{\square}$ & $6 / 19$ & $5 \times 5$ & $50 \times 50$ & $30 \times 30$ & $25 \times 60$ & $1,333 / 2,630$ & 20310 \\
\hline $6-5,5-\boldsymbol{\square}$ & $6 / 19$ & $5,5 \times 5,5$ & $50 \times 50$ & $30 \times 30$ & $29 \times 75$ & $1,364 / 2,989$ & 25640 \\
\hline $6-5-\square$ & $6 / 19$ & $5 \times 5$ & $60 \times 50$ & $40 \times 30$ & $25 \times 60$ & $1,206 / 2,480$ & 20785 \\
\hline $6-5,5-$ & $6 / 19$ & $5,5 \times 5,5$ & $60 \times 50$ & $40 \times 30$ & $29 \times 90$ & $1,241 / 2,836$ & 26115 \\
\hline
\end{tabular}




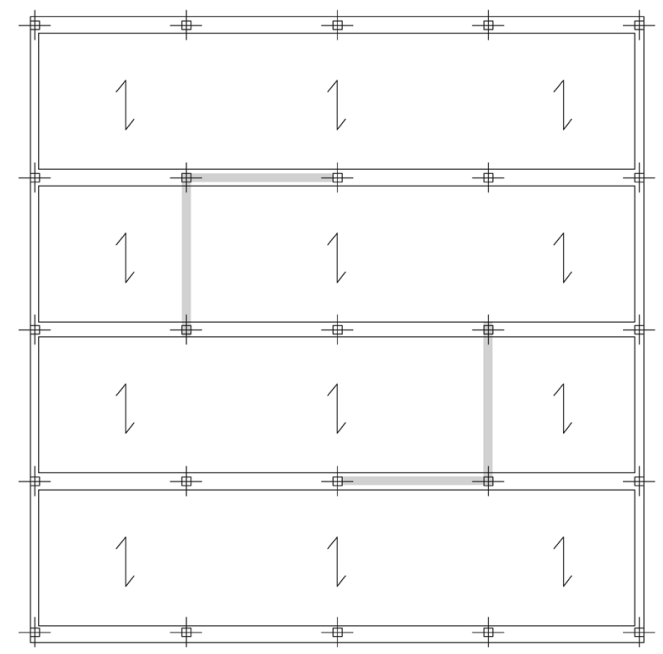

(a) “Muros mín.”

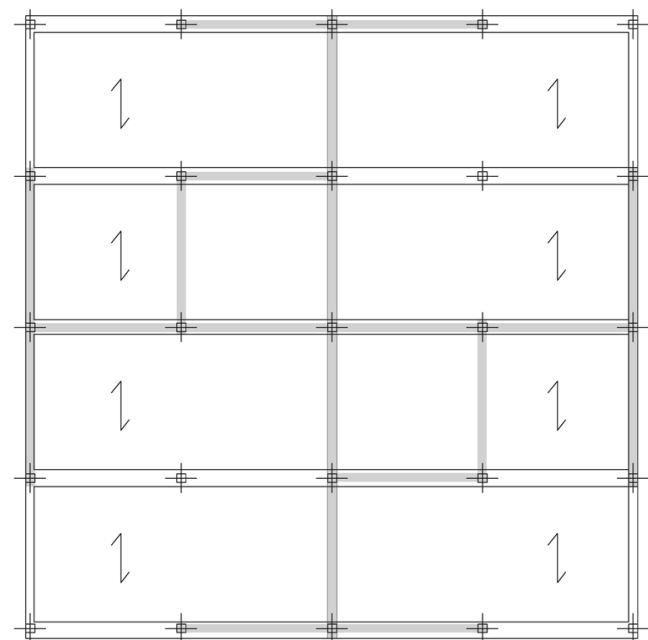

(b) “Muros máx.”

Figura 4. Ubicación de los muros en los edificios tipo.

En el trabajo (2) se describen en mayor profundidad las características de los edificios tipo.

\section{MODELACIÓN DEL COMPORTAMIENTO DINÁMICO DE LOS EDIFICIOS TIPO}

Este apartado describe el modelo del comportamiento estructural de los edificios considerados en los análisis dinámicos no lineales. Dichos análisis han sido realizados con el programa IDARC-2D en sus versiones 3.0 (11) y 7.0 (12).

\subsection{Modelización de la estructura principal}

Dada la simetría de los edificios tipo, su comportamiento estructural en cada dirección se representa mediante modelos planos. Cada modelo plano consta de los pórticos existentes en cada dirección; el efecto diafragma de los forjados se representa uniendo a estos pórticos con barras rígidas virtuales y biarticuladas a la altura de cada planta (2). Los pilares, las vigas y las viguetas están representados por elementos finitos de barra; las no-linealidades se concentran en rótulas plásticas situadas en las secciones próximas a los nudos. En los pórticos principales las uniones entre los pilares y las vigas planas se consideran rígidas; su comportamiento histerético (2) (13) se representa con modelos basados en los resultados experimentales disponibles (4). En dirección transversal las uniones entre los pilares y las viguetas se consideran rígidas para momentos negativos y articuladas para momentos positivos (dada la ausencia de armadura inferior de continuidad, Figura $3 \mathrm{~d}$ ); las uniones entre los pilares y las vigas de borde se consideran rígidas (Figura 3c). El comportamiento histerético de las uniones en ambas direcciones también se representa con modelos de fibras basados en las propiedades de los materiales, obteniéndose resultados similares.

\subsection{Modelización de los muros de relleno}

De forma previa, la influencia de muros de relleno de obra de fábrica en el período fundamental de los edificios se valora de una forma aproximada a partir de las expresiones contenidas en la normativa (14) (15). Dada la baja rigidez lateral de los edificios (especialmente en el plano ortogonal a los pórticos), se observa que incluso los rellenos más livianos generan una reducción significativa en el período fundamental; por ejemplo, con "muros mín." (cerramientos en núcleos de comunicación) en el edificio $3-5$ - (Tabla 1) el período fundamental en el plano en la dirección de los pórticos pasa de $0,585 \mathrm{~s}$ a $0,264 \mathrm{~s}$, siendo más significativa la disminución en el plano perpendicular donde el período pasa de 1,037 $\mathrm{s}$ a $0,285 \mathrm{~s}$. Esto confirma la importancia de los rellenos.

Se considera que la resistencia de los ladrillos es $f_{\mathrm{b}}=12 \mathrm{MPa}$ (16) y la del mortero es $f_{\mathrm{m}}=8 \mathrm{MPa}$; a partir de estos datos la resistencia característica $f_{\mathrm{bk}}$ y los módulos secantes de deformación longitudinal $E$ (10) y transversal $G$ toman los valores [1], [2].

$$
\begin{gathered}
f_{k}=K f_{b}^{0,70} f_{m}^{0,30}=0,45 \times 12^{0,70} \times 8^{0,30}=4,781 \mathrm{MPa} \\
E=500 f_{k}=2391 \mathrm{MPa} \quad G=0,4 E=956 \mathrm{MPa}
\end{gathered}
$$

El valor del coeficiente $K$ ha sido elegido teniendo en cuenta el tipo de ladrillo y la existencia de juntas longitudinales.

Los muros de relleno se representan por elementos finitos de barra que conectan entre sí las plantas adyacentes entre las que se sitúan los muros. Su comportamiento histerético se describe con los modelos de Bouc-Wen (17) (18); éstos consideran básicamente dos parámetros de los muros: la rigidez inicial y la resistencia. La rigidez inicial (elástica) de los muros se estima a partir de la formulación descrita en (19) como el doble de la rigidez final, calculando a ésta como el cociente entre la resistencia y el desplazamiento últimos. La resistencia y la rigidez (final) de los muros se determinan tal como se describe en (2) a partir del modelo de bielas y tirantes descrito en (19), ver la Figura 5. La altura (z) de las bielas en contacto con los pilares se determina a partir de las expresiones en (20) y el módulo equivalente de deformación se obtiene a partir de las recomendaciones de los Eurocódigos 8 y 6 (14) (15). Este estudio supone la conexión completa entre el muro y la estructura. El modo de fallo más crítico se determina entre los dos modos posibles: agotamiento por compresión de la diagonal comprimida y agotamiento por cortante de las juntas horizontales (tendeles). Las verificaciones descritas en (2) muestran que, en todos los casos considerados, el modo de fallo más crítico corresponde a compresión de la diagonal comprimida. No obstante, debe subrayarse que no se han capturado algunos efectos locales de las fábricas como el fallo 


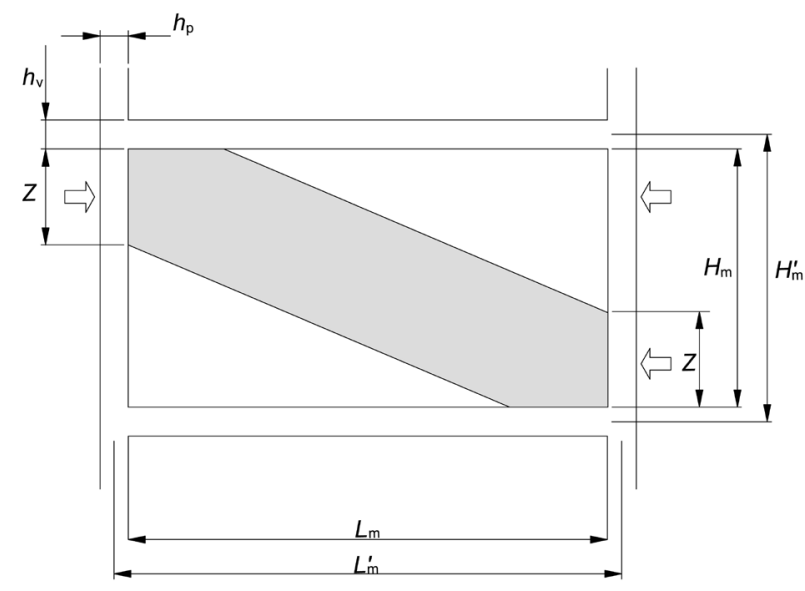

Figura 5. Modelo de bielas y tirantes de un muro de relleno.

a cortante de los pilares o el vuelco de las fábricas fuera de su plano, en especial de las de dos hojas.

\subsection{Análisis dinámicos}

Los cálculos dinámicos no lineales se llevan a cabo en tiempo discreto mediante el método b de Newmark (21). El período de discretización temporal $(\Delta t)$ se ha tomado igual al período de muestreo de los registros (o,001 s). El amortiguamiento de la estructura se representa mediante un modelo de Rayleigh (22), en el que el factor de amortiguamiento es igual al 5\%.

\section{RESPUESTAS DINÁMICAS DE LOS EDIFICIOS TIPO PARA LOS REGISTROS DEL TERREMOTO DE LORCA}

La Figura 6, la Figura 7 y la Figura 8 representan las historias temporales de las respuestas de los desplazamientos de las plantas superiores de, respectivamente, los edificios

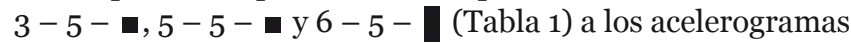

del terremoto de Lorca mostrados en la Figura 1; para los edificios de 5,5 m de luz, los resultados son semejantes. En las figuras xa y xb la componente norte-sur actúa en dirección de los pórticos mientras que en las figuras xc y xd dicha componente actúa en dirección transversal. Las curvas representadas en la Figura 6 a la Figura 8 se han interrumpido en el momento en que los desplazamientos de la planta superior han alcanzado los valores correspondientes al colapso según expresan las curvas de capacidad determinadas en los trabajos (2) (13).

Las respuestas mostradas en la Figura 6 a la Figura 8 señalan que, en prácticamente todos los casos, los edificios tipo colapsan en ambas direcciones. A este respecto debe tenerse presente que los análisis planos efectuados no permiten tomar en consideración la interacción de los movimientos en las dos direcciones; ello indica que la situación real será probablemente aun más desfavorable. Tan sólo los edificios de tres plantas (Figura 6) resisten la acción de la componente menos severa (la este-oeste, ver la Figura 1) del terremoto de Lorca si se toma en cuenta la cooperación de los muros (muros máx.).

La Figura 9 muestra las rótulas en los edificios $3-5,5-$ y 6 - 5,5 - - sin muros en el momento del colapso. Los esquemas representados se refieren a la dirección principal (de las vigas planas) bajo la actuación de la componente este-oeste (la menos severa, ver la Figura 1). En la Figura 9, $D$ es el índice de daño de Park y Ang (23). Los esquemas representados en la Figura 9 muestran que la mayor parte de rótulas plásticas y de roturas (es decir, de rótulas "completas") aparecen en los pilares, lo cual indica un mecanismo frágil de colapso. En el edificio de tres plantas (Figura 9a) las rótulas se concentran especialmente en las plantas superiores, mientras que en el edificio de seis plantas (Figura 9b) las rótulas se concentran especialmente en las plantas inferiores; ello indica un comportamiento aun más deficiente.

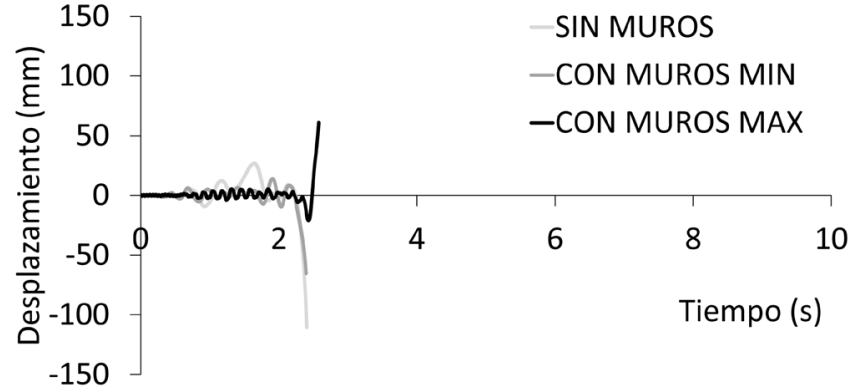

(a) Componente NS. Dirección de los pórticos

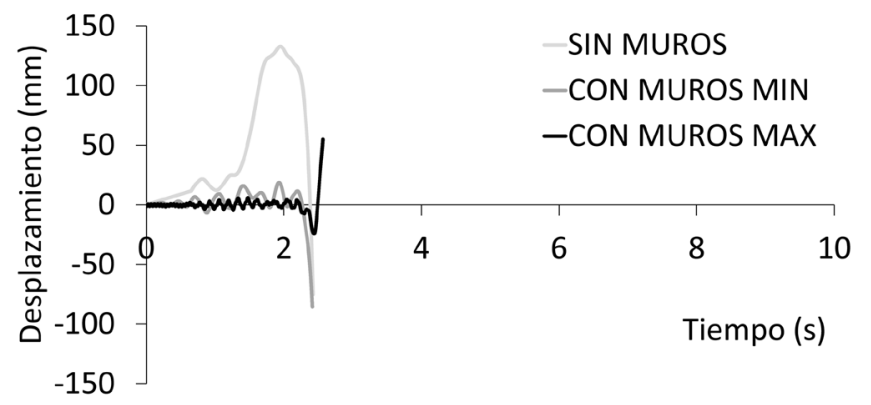

(c) Componente NS. Dirección transversal

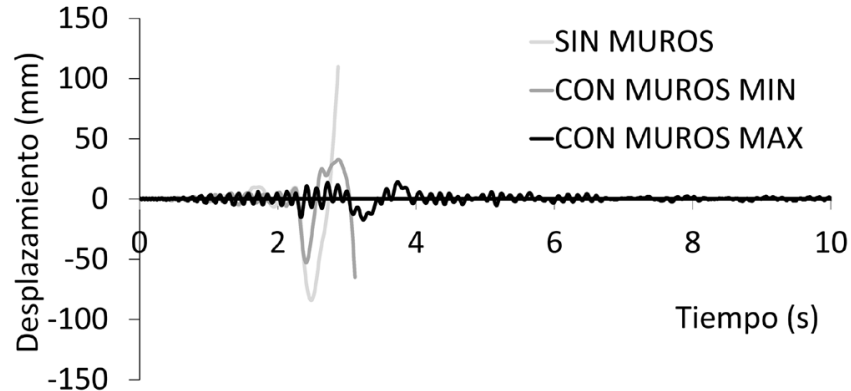

(b) Componente EW. Dirección de los pórticos

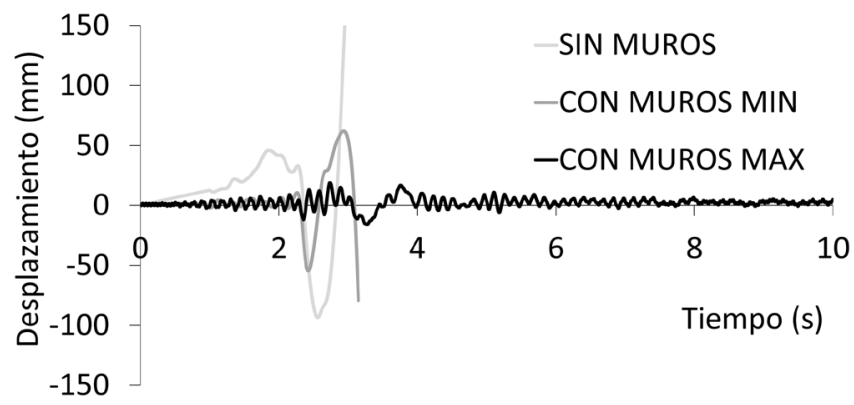

(d) Componente EW. Dirección transversal

Figura 6. Respuesta del edificio 3 - 5 - al acelerograma de Lorca (11 de mayo de 2011). 


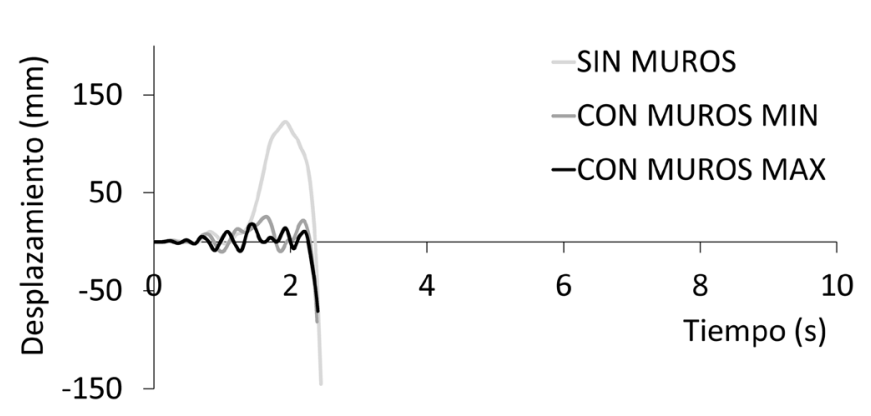

(a) Componente NS. Dirección de los pórticos

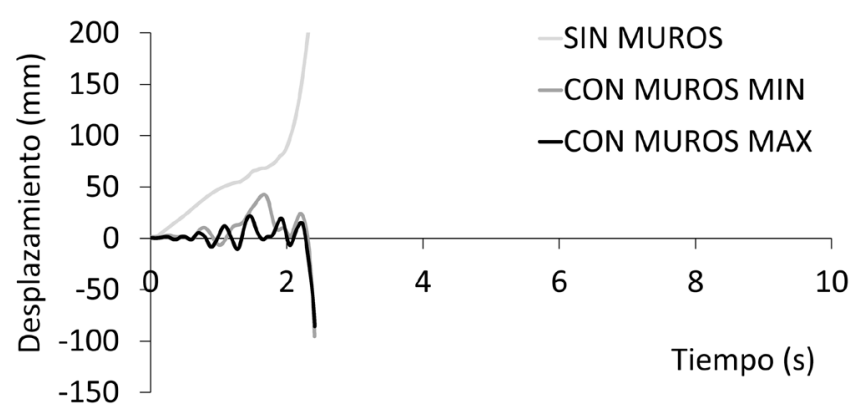

(c) Componente NS. Dirección transversal

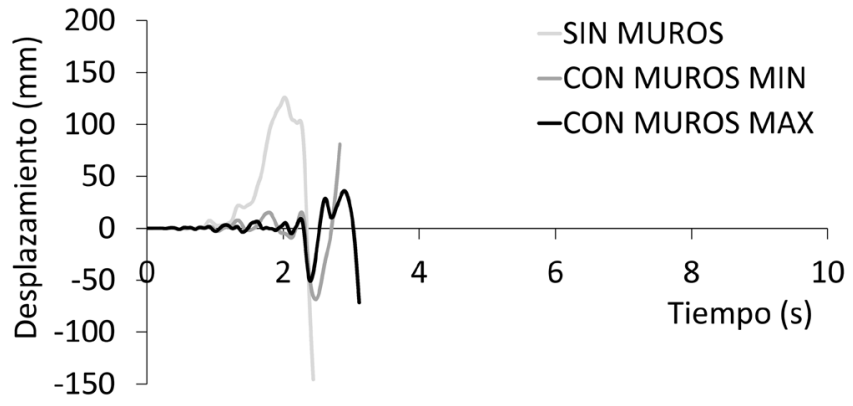

(b) Componente EW. Dirección de los pórticos

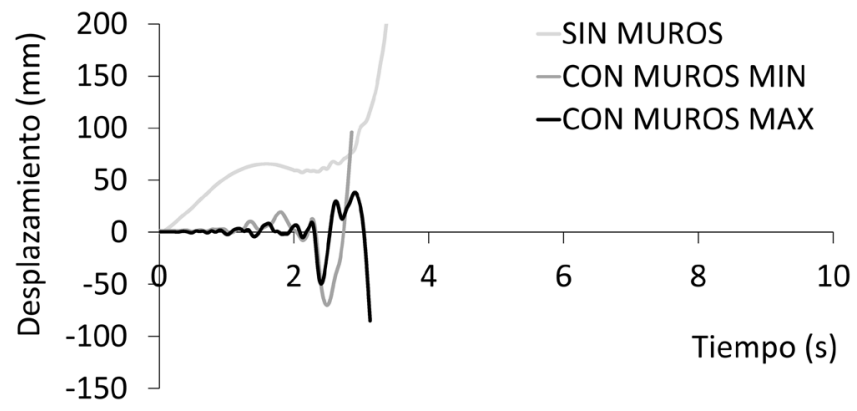

(d) Componente EW. Dirección transversal

Figura 7. Respuesta del edificio 6 - 5 - a al acelerograma de Lorca (11 de mayo de 2011).

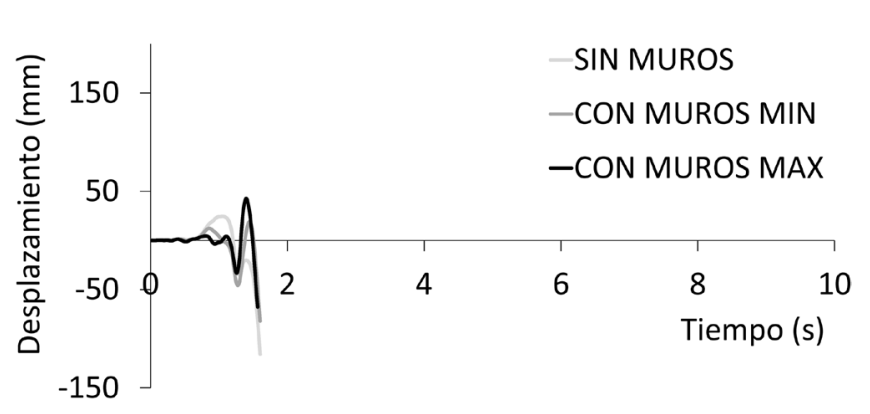

(a) Componente NS. Dirección de los pórticos

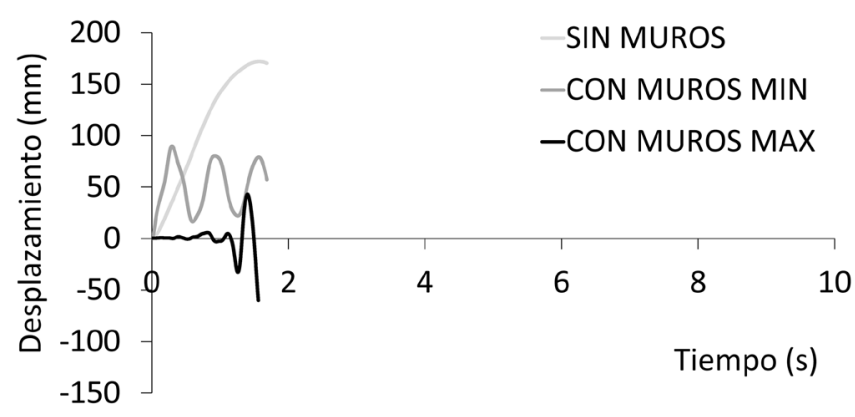

(c) Componente NS. Dirección transversal

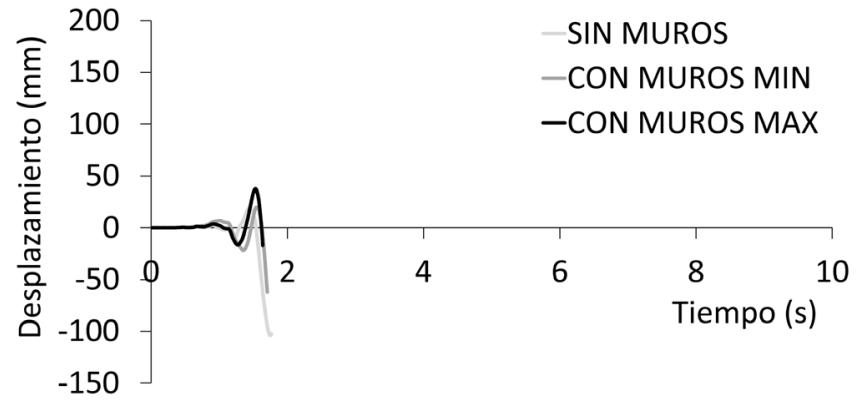

(b) Componente EW. Dirección de los pórticos

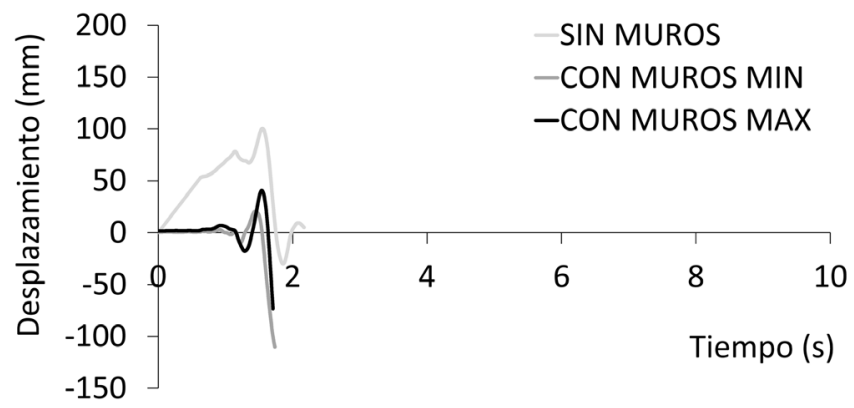

(d) Componente EW. Dirección transversal 


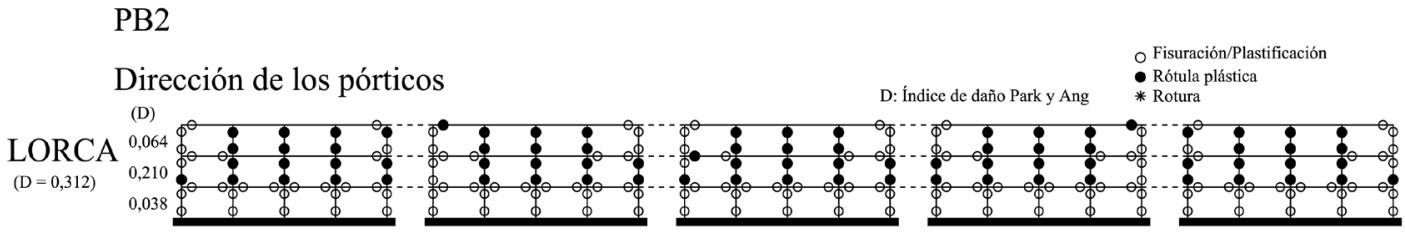

(a) Edificio 3 - 5,5 - - . Dirección de los pórticos

PB4

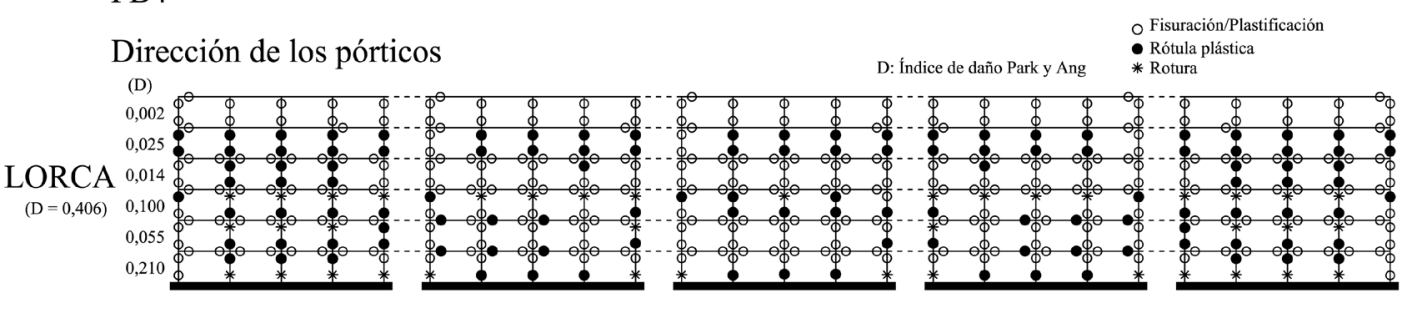

(b) Edificio 6-5,5 - a. Dirección de los pórticos

Figura 9. Daños en el momento del colapso.

\section{CONCLUSIONES}

Este trabajo presenta un estudio numérico acerca de la respuesta dinámica al reciente terremoto de Lorca (11-05-2011) de seis edificios de vigas planas de tres y de seis plantas, proyectados sin tener en cuenta la acción sísmica. Estos edificios pretenden representar a parte de los existentes. Los resultados obtenidos muestran que los edificios considerados, aun contando con la cooperación de los muros de relleno de obra de fábrica carecen de capacidad para resistir el terremoto de Lorca.

\section{AGRADECIMIENTOS}

Este trabajo ha sido financiado por el gobierno de España, proyectos CGL2008-00869/BTE, CGL2011-23621, BIA200800050 y BIA2011-26816 y por la Unión Europea (fondos Feder).

\section{REFERENCIAS}

(1) MOPU. (1974). PDS.1 (Normativa Sismorresistente). Ministerio de Obras Públicas y Urbanismo.

(2) Domínguez, D. (2012). Evaluación de la capacidad sismorresistente de edificios con vigas planas situados en zonas de España de sismicidad baja a moderada (Tesis Doctoral). Barcelona. Universidad Politécnica de Cataluña.

(3) MOPU. (1991). NBE-FL-9o (Norma Básica de la Edificación): Muros Resistentes de Fábrica de Ladrillo. Ministerio de Obras Públicas y Urbanismo.

(4) Benavent-Climent, A., Cahís, X., Vico, J.M. (2009). Interior wide beam-column connections in existing RC frames subjected to lateral earthquake loading. Bulletin of Earthquake Engineering, 8(2): 401-420, doi: http://dx.doi.org/10.1007/ s10518-009-9144-3.

(5) Murphy, P. (2011). Terremoto de Lorca 12 mayo 2011. Mesa redonda del 4 o Congreso nacional de Ingeniería sísmica. Granada.

(6) IGME. (2011). Informe geológico preliminar del terremoto de Lorca del 11 de mayo del año 2011, 5.1 Mw. Instituto Geológico y Minero de España.

(7) IGN. (2011). Serie terremoto NE Lorca (Murcia) 11/o5/2011. Instituto Geográfico Nacional.

(8) NCSE-02. (2002). Norma de Construcción Sismorresistente. Ministerio de Fomento.

(9) Asteris, P., Antoniou, S., Sophianopoulos, D., Chrysostomou, C. (2011). Mathematical Macromodeling of Infilled Frames: State of the Art. Journal of Structural Engineering, ASCE, 137(12): 1508-1517. doi: http://dx.doi.org/10.1061/ (ASCE)ST.1943-541X.0000384.

(10) Martínez, J.L., Martín, J.A., León, J. (2001). Comportamiento mecánico de la obra de fábrica. Monografía sobre el análisis estructural de construcciones históricas de fábrica. Madrid. Universidad Politécnica de Madrid.

(11) Kunnath, S.K., Reinhorn, A.M., Lobo, R.F. (1992). IDARC Version 3.o. A Program for the Inelastic Damage Analysis of Reinforced Concrete Structure, NCEER-92-0022. Buffalo. National Center for Earthquake Engineering Research - State University of New York.

(12) Kunnath, S.K., Reinhorn, A.M. (2010). IDARC 2D Version 7.o. Program for the Inelastic Damage Analysis of Reinforced Concrete Structure. Buffalo: State University of New York.

(13) López-Almansa, F., Domínguez, D., Benavent-Climent, A. (2013). Seismic performance of RC buildings with wide beams. Engineering Structures, 42(1): 687-702, doi: http://dx.doi.org/10.1016/j.engstruct.2012.08.033.

(14) EN 1998 (Eurocódigo 8). (2005). Design of Structures for Earthquake Resistance.

(15) EN 1996 (Eurocódigo 6). (2005). Proyecto de estructuras de albañilería.

(16) CTE DB SE-F. (2006). Seguridad Estructural: Fábrica. Ministerio de la Vivienda.

(17) Bouc, R. (1967). Forced vibration of mechanical systems with hysteresis. En Proceedings of the $4^{\text {th }}$ Conference on Nonlinear Oscillations, p.315, Prague, Czech Republic.

(18) Baber, T.T., Noori, M.N. (1985). Random Vibration of Degrading Pinching Systems. Journal of Engineering Mechanics, 111(8): 1010-1026, doi: http://dx.doi.org/10.1061/(ASCE)0733-9399(1985)111:8(1010). 
(19) Mostafaei, H., Kabeyasawa, T. (2004). Effect of Infill Masonry Walls on the Seismic Response of Reinforced Concrete Buildings Subjected to the 2003 Bam Earthquake Strong Motion: A Case Study of Bam Telephone Center. Bulletin of the Earthquake Research Institute, 79: 133-156.

(20) Paulay, T., Priestley, M.J.N. (1992). Seismic Design of Reinforced Concrete and Masonry Buildings. Wiley.

(21) Newmark, N.M. (1959). A method of computation for structural dynamics. Journal of Engineering Mechanics, ASCE, 85 (EM3): 67-94.

(22) Chopra, A.K. (2001). Dynamics of structures: theory and applications to earthquake engineering. Prentice Hall.

(23) Park, Y.J., Ang, A.H. (1985). Mechanistic seismic damage model for reinforced concrete. Journal of Structural Engineering (ASCE). 111(4): 722-739. 